\title{
Convergence of the study on monochromatic $X$-rays and the research on nanoparticles opens up a possibility to develop a new type of radiation therapy
}

\author{
Fuyuhiko Tamanoi ${ }^{1,2 *}$, Kotaro Matsumoto ${ }^{1}$, Tan Le Hoang Doan ${ }^{3}$, Ayumi Shiro ${ }^{4}$ and Hiroyuki \\ Saitoh $^{4}$ \\ 1 Institute for Integrated Cell-Material Sciences, Institute for Advanced Study, Kyoto University, Kyoto, \\ Japan; tamanoi.fuyuhiko.2c@kyoto-u.ac.jp \\ 2 Dept. of Microbio., Immunol. \& Molec. Genet., University of California, Los Angeles, Los Angeles, CA, \\ USA \\ 3 Center for Innovative Materials and Architectures (INOMAR), Vietnam National University-Ho Chi Minh \\ City, Ho Chi Minh City, Vietnam \\ 4 Kansai Photon Science Institute, Quantum Beam Science Research Directorate, National Institutes for \\ Quantum and Radiological Science and Technology, Hyogo, Japan \\ * Correspondence: tamanoi.fuyuhiko.2c@kyoto-u.ac.jp, Tel.: 81-75-753-9856
}

\begin{abstract}
Conventional radiation therapy uses white $\mathrm{X}$-rays that consist of a mixture of $\mathrm{X}$-ray waves with various energy levels. In contrast, a monochromatic $X$-ray (monoenergetic $X$-ray) has a single energy level. Irradiation of high $\mathrm{Z}$ elements with a synchrotron generated monochromatic X-ray with the energy at or higher than the K-edge energy of the element results in the production of the Auger electrons that cause DNA damage leading to cell killing. Delivery of high Z elements into cancer cells and tumor mass can be facilitated by the use of nanoparticles. Mesoporous silica nanoparticles (MSNs) have been shown to be effective in delivering high $\mathrm{Z}$ elements to cancer cells. A proof of principle experiment was reported that demonstrated the feasibility of this approach. This opens up a possibility to pursue the Auger cancer therapy by the combined use of MSNs loaded with high $\mathrm{Z}$ elements and monochromatic X-rays. Similar cancer therapies using other types of quantum beams such as neutron, proton and carbon ion beams can be envisioned.
\end{abstract}

Keywords: monochromatic X-ray, mesoporous silica nanoparticles, high Z elements, K-edge energy

\section{Introduction}

Nanotechnology that was initiated in 1960s has generated a variety of nanomaterials valuable for biomedical applications such as cancer therapy [1]. Of particular interest are nanoparticles, small particles of the size ranging from 40 to $400 \mathrm{~nm}$ [2-4]. Various materials have been used to generate nanoparticles including organic nanoparticles such as liposomes, synthetic polymers, micelles, protein and biomolecules as well as inorganic nanoparticles such as mesoporous silica nanoparticles, gold nanoparticles and diamond nanoparticles [2-4]. Many nanoparticles are efficiently taken up into cancer cells by endocytosis and can accumulate in late endosomes and lysosomes that are localized at the perinuclear region of a cell [5]. In addition, nanoparticles can accumulate in the tumor either via passive enhanced permeability and retention (EPR) mechanism as well as by active targeting mechanisms [2,6].

Convergence of Nanotechnology and Radiation therapy promises to result in the generation of various new approaches to radiation therapy $[7,8]$. For example, radiosensitizers have been delivered by nanoparticles to enhance radiation therapy [7]. Advantageous properties of nanoparticles such as tumor targeting have important implication for radiation therapy. Another approach that has 
recently emerged is to deliver high Z elements by nanoparticles into tumor mass and to irradiate with a monochromatic X-ray beam [9]. In this study, gadolinium was loaded onto nanoparticles and distributed throughout tumor spheroids. Irradiation of the spheroids with a monochromatic X-ray having the energy corresponding to the K-edge energy of gadolinium resulted in efficient destruction of the tumor mass. A key observation was that the destruction was sharply dependent on a particular energy level. This opens up the possibility of developing the Auger cancer therapy by the use of monochromatic X-rays and high Z element loaded nanoparticles.

In this review, we first discuss special properties of monochromatic $X$-rays and then discuss the Auger effect that was proposed in 1923 [10]. We describe nanoparticles that can be used to deliver high $\mathrm{Z}$ elements and discuss how nanoparticles can enhance the Auger effect in cancer cells. Related work that uses the general scheme of combining radiation beams and nanomaterials will be discussed. Because various beams such as proton, neutron and carbon ion beams are used widely in biomedical applications [11], convergence of quantum beams with nanomaterials containing various elements may open up a new field of study concerning radiation therapy.

\section{Monochromatic X-ray}

2.1 White X-ray and monochromatic X-ray

$X$-rays are electromagnetic waves in the wave length range of $1 \mathrm{pm}$ to $10 \mathrm{~nm}$ (Figure 1A) [12]. Current radiation therapy uses white $\mathrm{X}$-ray which is a mixture of these $\mathrm{X}$-rays waves. Because of this

\section{A}

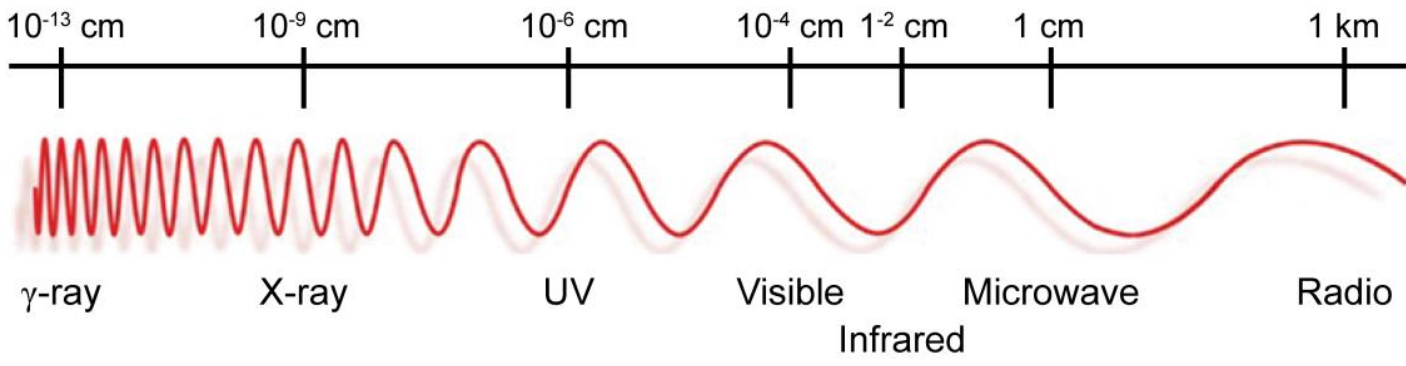

B

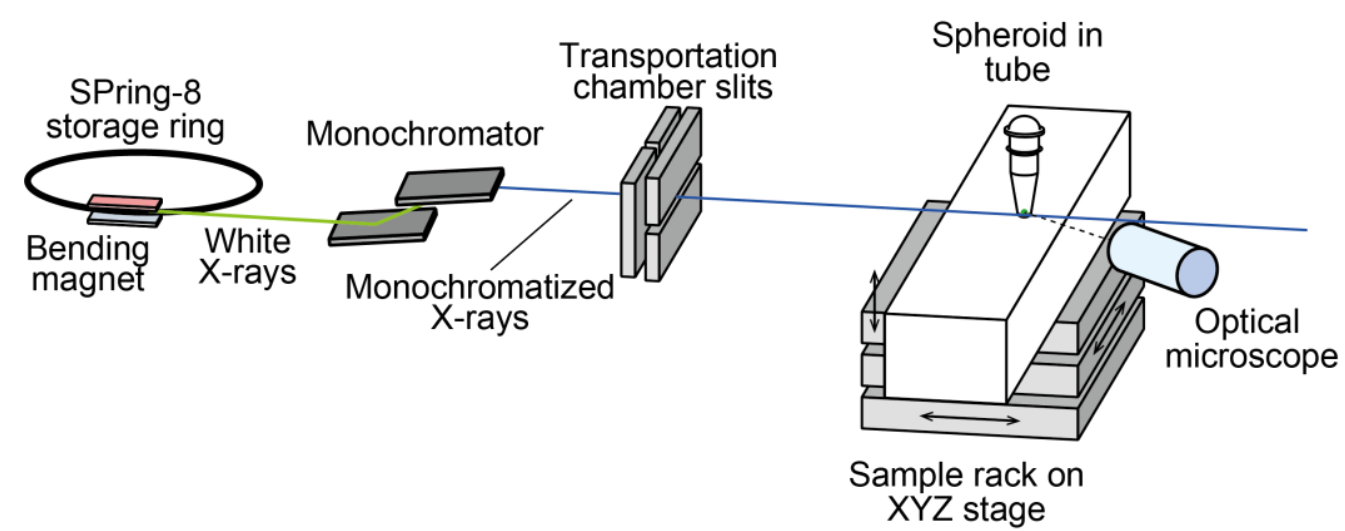

Figure 1. A: Various types of electromagnetic waves. Wave length is shown above the line. B: Synchrotron generated electron beams (white X-rays) are separated into monochromatic X-ray beams by a monochromator. This is used to irradiate tumor spheroids as described in the text. Modified from [9]. 
feature, conventional X-rays interact with various atoms in the human body. For example, energy from X-ray will be absorbed by various atoms on the skin causing skin damage including sunburn like symptoms. In addition, conventional radiation causes effect on tissues other than the target tumor.

Similar to the use of a prism that separates various color waves in the visible light range, it is possible to separate conventional $\mathrm{X}$-rays into monochromatic $\mathrm{X}$-ray waves each having a single energy level by using a monochromator (Figure 1B) [13,14]. The amount of X-ray absorption by material depends on the kind of irradiated elements and the energy of $X$-rays. For example, when a monochromatic X-ray of $50.25 \mathrm{keV}$ is used, energy is absorbed specifically by gadolinium but not by other elements. Therefore, elements in the human body such as carbon, oxygen, hydrogen, phosphate, nitrogen will not be affected by this monochromatic X-ray beam. This lack of interaction with biological materials is a key advantage of the monochromatic X-ray. Monochromatic X-rays have been used for imaging studies $[15,16]$. Lack of interaction with various elements contributes to reducing the background images that are seen with conventional X-ray imaging methods. Thus, much sharper images can be obtained.

\subsection{Synchrotron generated monochromatic X-ray}

Currently, the best source for monochromatic X-ray is to use synchrotrons that generate intense X-ray beams. A synchrotron accelerates electrons to extremely high energy and then direct them to change directions by the use of magnets (bending magnets). When a moving electron changes direction, they emit energy at X-ray wavelength. The X-ray beams emitted from a bending magnet are directed to the beamlines that are placed surrounding the synchrotron ring.

In our study, SPring-8 synchrotron radiation facility located in Harima, Japan, was used (Figure 1B). The storage ring is operated with a constant storage ring current of $100 \mathrm{~mA}$ [9]. The monochromatic $X$-rays from a bending magnet were shaped by horizontal and vertical slits. The incident beam size is $1.4 \mathrm{~mm}$ in height and $1.4 \mathrm{~mm}$ in width at the sample position [9].

\section{The Auger effect}

In 1923, Pierre Auger reported at the French Academy of Science that the irradiation of high Z element with monochromatic X-rays results in the release of electrons (Figure 2) [10]. For example, when gadolinium was irradiated with the monochromatic X-ray having a higher energy than the Kedge energy of gadolinium, an electron in the K-shell will absorb the energy and the electron is kicked out of the atom. This results in the destabilization of the atom which is corrected by having an electron from outer shell drop to the K-shell. This releases energy which is then used to kick out other electrons from the atom. Thus, the series of events lead to the release of electrons. If an element other than gadolinium is used, then the monochromatic X-ray energy needs to be adjusted so that it is higher than the K-edge energy of the particular element (discussed further in section 6). The electrons released are called the Auger electrons that possess strong cell killing effect including ionization and cleavage of DNA. However, they travel only a short distance and thus the effect is confined within the cancer cell [17-20]. Occurrence of these electrons was reported in 1922 by Lise Meitner (discussed in reference 17). 
Thus, the effect of monochromatic X-rays can be amplified by the use of high Z elements, raising the possibility that the Auger effect-based cancer therapy can be developed. There has been a quest to develop the Auger therapy (reviewed in ref. [17-20]). In early studies, biological effect of ${ }^{125 I-}$ nucleotides incorporated into DNA was examined, as they undergo natural decay resulting in the release of Auger electrons. The study uncovered cell killing property including DNA cleavage. Subsequently, photon-activation therapy (PAT) was examined. However, extent of photon induced

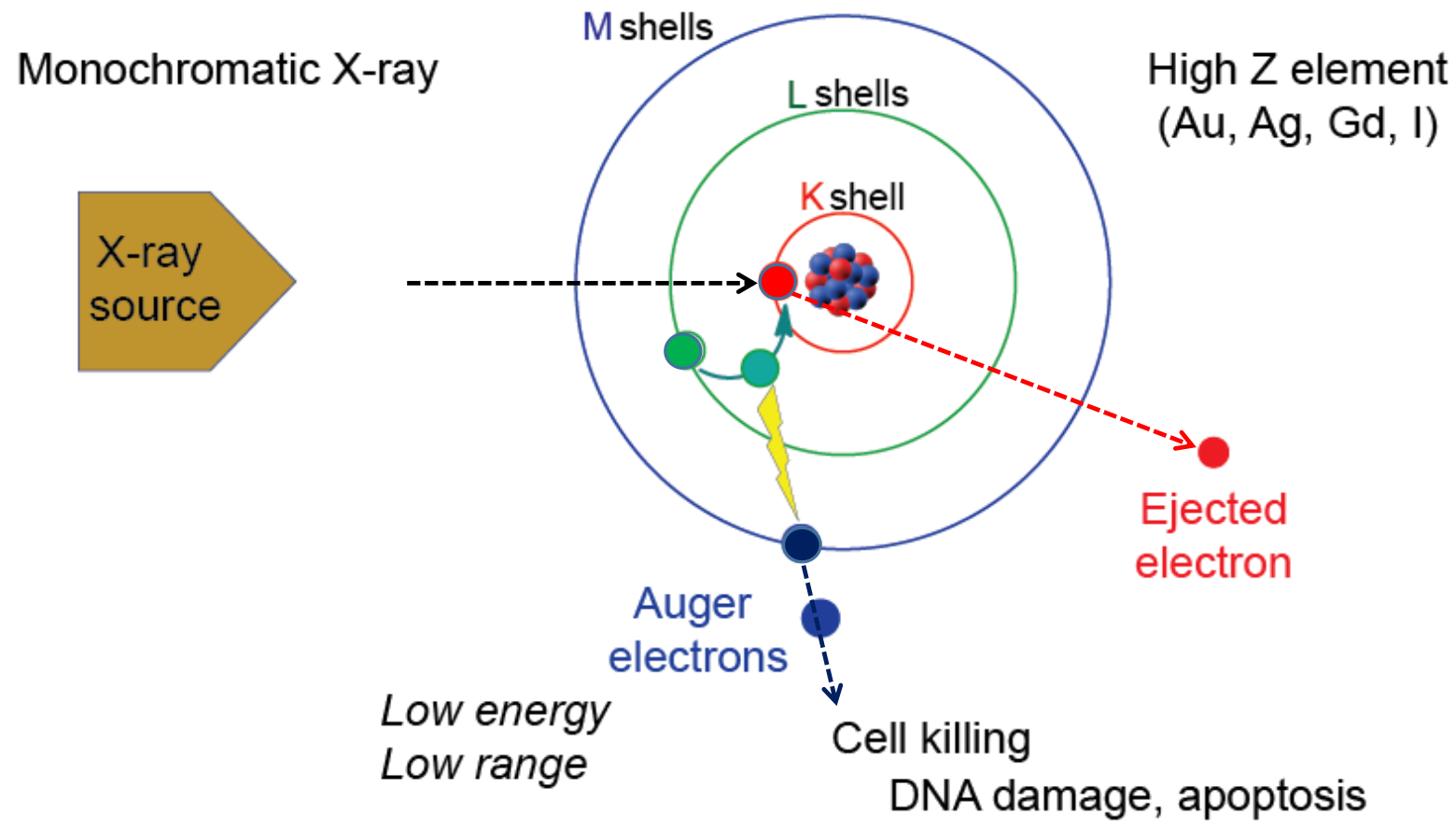

Figure 2. The Auger effect. Irradiation of high Z element such as Au, Ag, Gd or I with a monochromatic Xray having a defined energy level results in the absorption of a K-shell electron (red circle). This results in the movement of an electron from a higher shell (green circle) causing the release of energy that is used by another electron (blue circle) which is released as an Auger electron. The Auger electron has strong cell killing effect.

effect examined by using cancer cells as well as mouse models was not strong [17]. One of the reasons for the failure may be due to low cytoplasmic concentration of the high $\mathrm{Z}$ element [17]. This is where nanoparticles can make a big difference as discussed below.

\section{Nanoparticles}

Advance in Nanotechnology led to the development of various nanomaterials such as carbon nanotubes, fullerene and quantum dots that provide valuable materials for medical therapy and diagnosis. In particular, nanoparticles, tiny particles of the size range 40-400 nm are important for drug delivery and a variety of nanoparticles have been developed over the years [2,3]. These include liposomes, synthetic polymers including polymer micelle. Inorganic nanoparticles such as silica, silicon, gold and diamond, also have been developed. In addition, virus nanoparticles and protein nanoparticles have been developed.

Among various nanoparticles, mesoporous silica nanoparticles (MSN) have recently emerged as a powerful and versatile material [21-25]. They are synthesized by the sol-gel method that involves condensation of TEOS (tetraethyl orthosilicate) in the presence of templating surfactant solution. 
Figure 3 shows MSN nanoparticles we used for biomedical application [26]. While these nanoparticles have approximately $100 \mathrm{~nm}$ diameter, the size can range from 50-400 nm by changing synthesis conditions. MSN shown in the figure contains 1400 pores with each pore diameter in the range of 2-4 $\mathrm{nm}$ giving the appearance of honey comb or Swiss cheese.

The key feature of MSN is the presence of a vast surface area. Because the pore interior can be considered surface, this type of nanoparticles has a huge surface where various compounds can be attached; it has been estimated that $1 \mathrm{~g}$ of MSN has $100 \mathrm{~m}^{2}$ of surface area which translates to an area of $10 \mathrm{~m} \times 10 \mathrm{~m}$ [4]. The chemistry for grafting various functional groups on the surface has been established [4]. This uses APTES with a variety of functional groups including amines, phosphonates, sulfates etc. Hydrophobic surface can also be prepared. Various size nanoparticles with various surface charges can be synthesized.

A

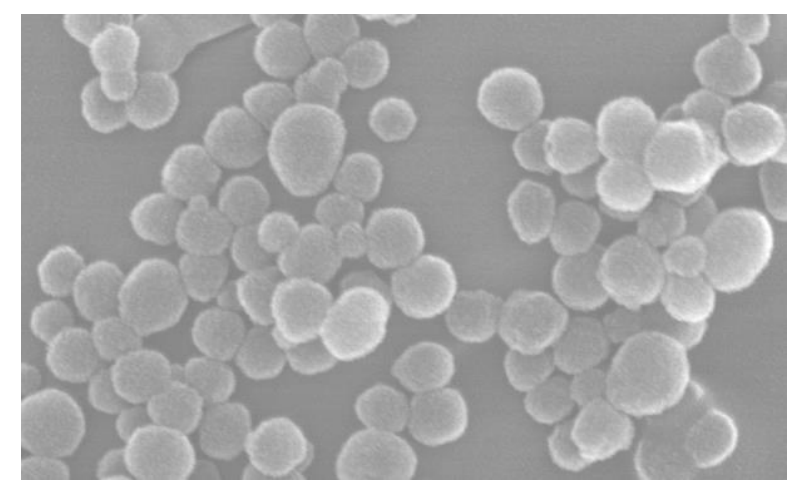

$100 \mathrm{~nm}$
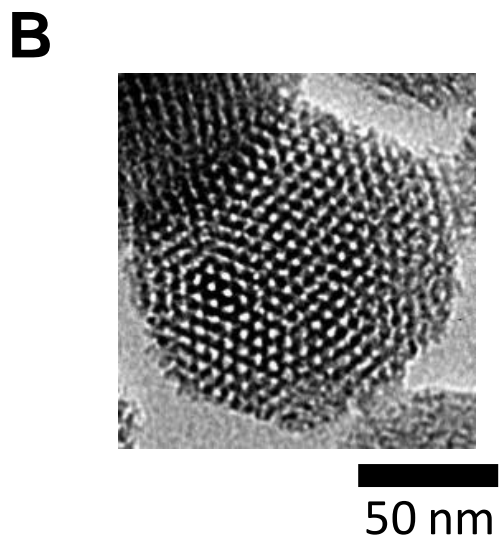

Figure 3. Mesoporous silica nanoparticles prepared by the sol-gel method. A: SEM picture. B: TEM picture. Modified from [9].

MSN nanoparticles are biocompatible and safe [27]. Recently, biodegradable MSN have been developed by incorporating biodegradable bonds into the framework of the nanoparticle [28-32]. These nanoparticles are called biodegradable periodic mesoporous organosilica (BPMO) and the synthesis involved the use of bridged alkoxysilane precursor instead of TEOS used for the synthesis of MSN. The bridged alkoxysilane is made up of two silane units bridged by a chemical bond. The biodegradable chemical bonds that have been used include di- and tetrasulfide bonds that are cleavable by reducing conditions such as those encountered inside the cell. In addition, protease sensitive bonds have been used. Degradation in vitro of BPMO and delivery of anticancer drugs by BPMO has been reported [28].

\section{Proof-of-principle experiments for the Auger cancer therapy using gadolinium-loaded MSN}

We have recently provided a demonstration that destruction of tumor mass can be achieved by incubating tumor spheroids with gadolinium loaded MSN and irradiating with a monochromatic Xray [9]. We used a monochromatic X-ray of $50.25 \mathrm{keV}$ (just above the K-edge absorption energy). To do this, we first synthesized gadolinium loaded MSN. MSN nanoparticles were surface modified so that they have excellent dispersibility in a solution. One of the ways to accomplish this is to use 
phosphonate to provide negative charge to the nanoparticle surface [33]. Gadolinium was attached to MSN by first preparing amine modified MSN by using APTES and then incubated with gadopentetic acid to prepare Gd-MSN [9]. Once bound, gadolinium is stably associated with MSN.

As for the tumor mass, we opted to use tumor spheroids that are three-dimensional structure of cancer cells prepared by using a special plate for growing cancer cells (Figure 4A) [34]. It is believed that the three-dimensional tumor represents human tumor better than two-dimensional tissue

\section{A: Distribution of Gd-loaded MSN throughout tumor a spheroid}

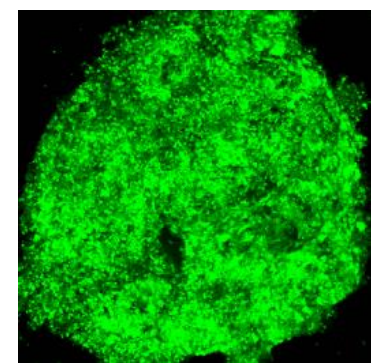

Tumor spheroid of GFP-cancer cells

\section{Rhodamine-B labeled Gd-MSN}

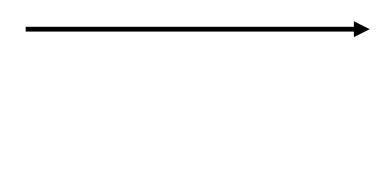

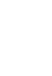

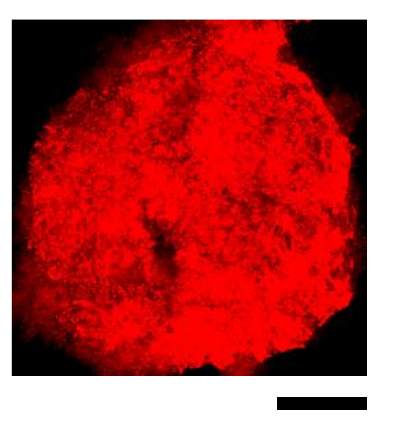

$100 \mu \mathrm{m}$

\section{B: Intracellular localization of Gd-MSN}
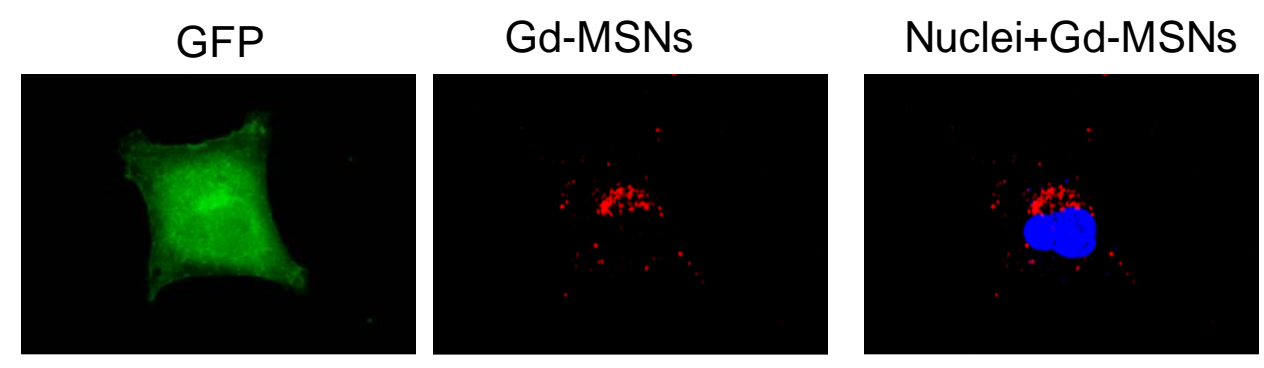

Figure 4. A: Tumor spheroid prepared from GFP-expressing cancer cells is shown as a green mass. The size of the spheroid is usually $0.3 \mathrm{~mm} \times 0.3 \mathrm{~mm}$. When the spheroid was incubated with gadolinium-loaded MSN labeled with Rhodamine- $\mathrm{B}$, uniform distribution of the nanoparticle throughout the spheroid was observed by using confocal microscopy (see the red fluorescence). B: Within a cell, Gd-MSN accumulates in a perinuclear region (nucleus is stained with Hoechst and appears blue). Modified from [9].

culture cells. In addition, the center of tumor spheroids often contains a necrotic area that again resembles human tumor. In fact, response to anticancer drugs is different with tumor spheroids compared with tissue culture cells. While tumor spheroids consist only of cancer cells, there has been effort to include other types of cells such as stromal cells to mimic tumor microenvironment. These are called tumor organoids $[35,36]$.

Gd-MSN can be distributed throughout tumor spheroids, as shown by confocal microscope analyses of tumor spheroids after incubation with red fluorescent MSN (Figure 4A). In this experiment, we used ovarian cancer OVCAR8 cells expressing GFP so that the tumor spheroid shows green fluorescence. Incubation with Rhodamine-B labeled red fluorescent MSN results in the distribution of red fluorescence on the tumor spheroid (Figure 4A). Overlap of green and red fluorescence was further confirmed by the examination of each focal plane of the tumor spheroid [9]. 
Figure 4B shows localization of Gd-MSN in the perinuclear region of a cancer cell. In this experiment, cell nucleus is stained with Hoechst dye which gives blue fluorescence. As can be seen, red fluorescence of Gd-MSN is detected just outside of the cell nucleus. This is because MSN can be taken up into cancer cells by energy-dependent endocytosis and localize to late endosomes and lysosomes that exhibit perinuclear localization [26]. Thus, MSN can be used to deliver various materials to the perinuclear region of a cell.

Monochromatic X-rays generated at SPring-8 synchrotron facility was used to irradiate the tumor spheroids that have been incubated with Gd-MSN (Figure 1B). This X-ray beam of $1.4 \mathrm{~mm} \times 1.4 \mathrm{~mm}$ was guided to the tumor spheroid sample and the irradiation continued for up to 1 hour. After the exposure, the spheroids were incubated for two to three days till the effect of the irradiation becomes apparent. As can be seen in Figure 5, we found that the exposure of the tumor spheroids resulted in almost complete destruction of the spheroids. Spheroid destruction was dependent on the exposure time but significant destruction was observed even after 10 minutes. Exposure of the tumor spheroids to the $50.25 \mathrm{keV}$ monochromatic X-ray did not cause destruction without the presence of gadolinium.

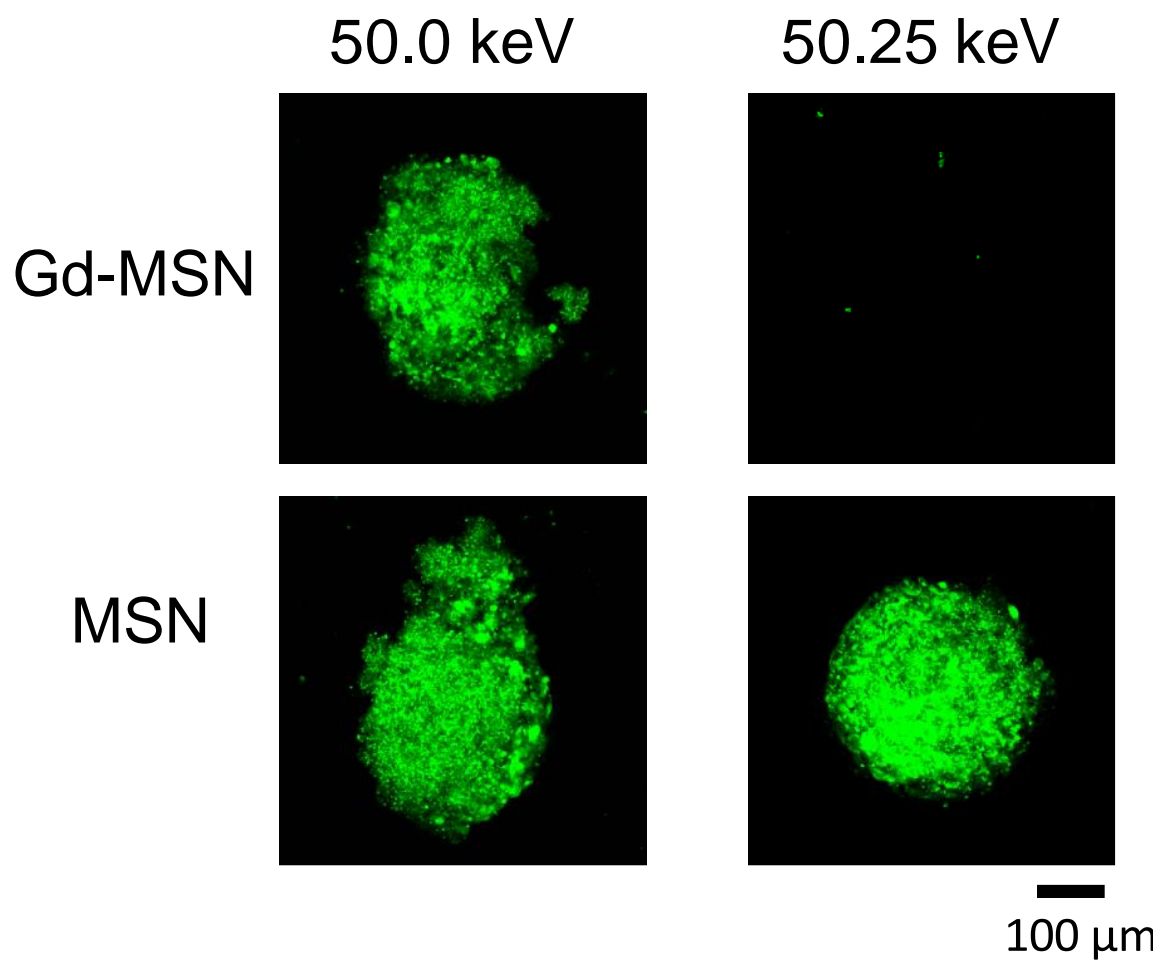

Figure 5. Tumor spheroid incubated with Gd-MSN was destructed after irradiation with a monochromatic X-ray of $50.25 \mathrm{keV}$ for 60 minutes and then incubated for three days. Tumor spheroid with empty MSN was not affected by the irradiation. Modified from [9].

Of particular importance is that the destruction of tumor spheroid was dependent on the use of a monochromatic X-ray with a particular energy level [9]. As shown in Figure 5, we used monochromatic X-rays of $50.0 \mathrm{keV}$ and $50.25 \mathrm{keV}$. This is based on a preliminary experiment examining energy absorption of gadolinium, which demonstrated a sharp increase in the energy absorption at $50.25 \mathrm{keV}$. As can be seen, almost complete destruction of tumor spheroid was observed when a monochromatic X-ray of $50.25 \mathrm{keV}$ was used. Destruction of tumor spheroid was also observed with the monochromatic X-ray having $50.4 \mathrm{keV}$ energy, although the destruction was 
somewhat less. In contrast, no destruction was observed when a monochromatic X-ray of $50.0 \mathrm{keV}$ was used. Thus, the tumor destruction occurs at $50.25 \mathrm{keV}$ that is just above the K-edge energy.

\section{The Auger cancer therapy.}

The results described above are consistent with the idea that the Auger electrons caused tumor mass destruction. As shown in Figure 6, it is possible to envision a cancer therapy using monochromatic X-ray and high Z element such as gadolinium. The key is to deliver these elements to the tumor and place them close to DNA. Use of nanoparticles such as MSN can be employed to deliver high $\mathrm{Z}$ element to the tumor site. Further work with animal model systems is needed to investigate the potential of the Auger therapy.

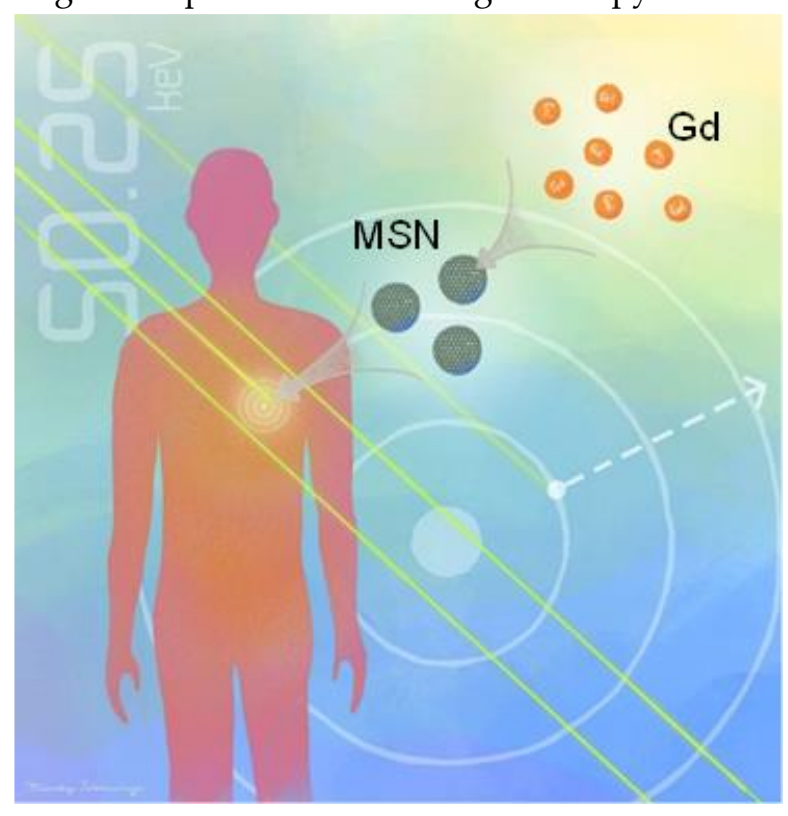

Figure 6. The Auger cancer therapy envisions loading of high $Z$ elements such as gadolinium onto nanoparticles such as MSN which will achieve selective accumulation of high $\mathrm{Z}$ elements in the tumor. Irradiation with a monochromatic $\mathrm{X}$-ray with a defined energy level promises to destruct tumor mass.

The key feature of the Auger cancer therapy is that the energy of monochromatic X-ray needs to be selected based on the K-edge energy of the high $\mathrm{Z}$ element used (Figure 7). For example, if gold is used, a monochromatic X-ray with $80.7 \mathrm{keV}$ needs to be used to generate the Auger electrons. On the other hand, elements such as iodine or silver can be used with monochromatic X-rays with lower energies such as 33.2 and $25.5 \mathrm{keV}$, respectively. X-rays with these energy levels may be generated using a compact synchrotron or even laser-guided X-ray sources [37]. The study on these elements may stimulate research on compact $\mathrm{X}$-ray sources. It has been reported that high $\mathrm{Z}$ elements such as gadolinium can absorb energy from conventional X-ray and this results in the enhancement of radiation therapy [38]. Similar studies have used iodine nanoparticles [39]. These studies emphasize the importance of high $\mathrm{Z}$ elements on enhancing conventional X-ray therapy. 


\section{High Z elements}
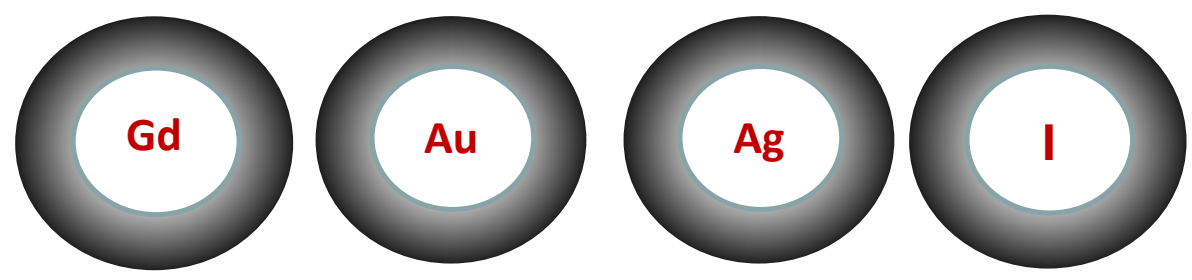

\section{K-edge energy}

\section{$50.2 \mathrm{keV} \quad 80.7 \mathrm{keV} \quad 25.5 \mathrm{keV} \quad 33.2 \mathrm{keV}$}

Figure 7. Various high Z elements and their K-edge energy.

\section{Use of neutron beam.}

Instead of X-rays, other types of beams are being used for medical therapy (Table 1). In the case of boron neutron capture therapy (BNCT), ${ }^{10} \mathrm{~B}$ (boron-10) is exposed to neutron beam, resulting in the splitting of boron atom to lithium and helium [40-43]. Helium nucleus is an $\alpha$-particle that has strong destructive effect including DNA cleavage. Clinical studies were initiated right after world war II and then more extensively in 1980s and 1990s [40,42]. The boron compounds used include BPA (boronophenylalanine) and BSH (sodium borocaptate). In clinical settings, these reagents are administered by intravenous injection into patients. Nanoformulated boron reagents such as boron10 loaded liposomes and polymers have been developed [44,45].

Exposure of gadolinium to neutron beam releases $\gamma$-ray as well as electrons leading to destruction of cancer cells. This method is called GNCT (gadolinium neutron capture therapy) and has been evaluated as an alternative to BNCT [46]. The main difference between BNCT and GNCT is that $\alpha$-ray is emitted from boron-10 in the case of BNCT, while $\gamma$-ray is emitted from gadolinium in the case of GNCT. Because $\alpha$-ray's travel path is more limited than $\gamma$-ray, effect on tumor is more focused with BNCT. However, further investigation is needed to compare BNCT and GNCT.

\section{Various quantum beams}

In addition to X-ray and neutron beams discussed above, there are other quantum beams that are used for cancer therapy. For example, proton beams are widely used as an effective cancer therapy [11]. This is because proton beams can be optimized so that the energy level at the site of tumor will be high, in contrast to X-ray that loses energy as it penetrates into the tissue. There are many proton therapy centers that are carrying out cancer therapy. This raises the possibility that the proton beam can be used to combine with various elements delivered to the tumor by the use of nanomaterials. 
The first demonstration of this possibility was reported by Cirrone et al [47]. In this work, proton

\section{Quantum beam Nanomaterials Cancer Therapy}

$\begin{array}{lll}\begin{array}{l}\text { Monochromatic } \\ \text { X-ray }\end{array} & \text { High Z element/NP } & \text { Auger therapy } \\ \text { Neutron } & \text { Boron-10/NP } & \begin{array}{l}\text { Boron neutron } \\ \text { capture therapy }\end{array} \\ \text { Neutron } & \text { Gadolinium/NP } & \begin{array}{l}\text { Gadolinium neutron } \\ \text { capture therapy }\end{array} \\ \text { Proton } & \text { Boron-11/NP } & \begin{array}{l}\text { Boron proton } \\ \text { capture therapy }\end{array}\end{array}$

Table 1. Various high Z elements and their K-edge energy and Auger yield

beam was irradiated on boron-11 in BSH (sodium borocaptate) resulting in the release of $\alpha$-rays (proton exposure of natural boron results in the release of three $\alpha$-particles). Cell death and DNA damage was observed with breast cancer cells. This therapy is called boron proton capture therapy (PBCT) and may turn out to be a powerful alternative to BNCT.

Another quantum beam that is widely used in cancer therapy is carbon ion beam. This beam has been used to ionize molecule inside cancer cells. Effects on cancer cells are reported to be more substantial and DNA damage appears to occur within a narrow region of DNA. At this point, no nanomaterials that can be combined with the carbon ion beam has been reported. Yet, another quantum beam is an electron beam that can be generated by various nuclear reactions. Irradiating various elements with an electric beam might be interesting, but we have yet to see a report on this point. Advance in the study of particle physics promises to generate various new quantum beams. Together with the development of various nanomaterials, it is possible to develop novel therapies that could greatly enrich the current X-ray therapy.

\section{Summary}

Monochromatic X-rays provide a valuable source for radiation therapy. These special type of Xray with a single energy level can be obtained by separating a synchrotron generated white X-ray by the use of a monochromator. High $\mathrm{Z}$ elements such as gadolinium can be irradiated with the monochromatic X-rays having energies higher than K-edge energies of the element resulting in the release of the Auger electrons. Occurrence of this reaction in cancer cells results in DNA damage and induction of cell killing. Proof of principle demonstration on this approach using gadolinium loaded MSN and monochromatic X-rays has been reported. This opens up a possibility of pursuing the Auger cancer therapy. This approach can be expanded to include other quantum beams such as neutron, proton and carbon ion beams.

Author Contributions: All authors have contributed to the conception, writing and discussion of this review. All authors have read and agreed to the publication of the manuscript. 
Funding: This work was supported by JSPS KAKENHI Grant No. JP15K21764 (to FT), by National Institutes for Quantum and Radiological Science and Technology (Proposal No 2018B3658, 2019A3658 and 2019B3658) (to HS) and by a grant from Vietnam National University Ho Chi Minh City grant number VNU-A-562-2018-50-01 (to TLHD).

Acknowledgments: We would like to thank the members of the Tamanoi Lab for discussion. We also acknowledge Mindy Takamiya for drawing Figure 6.

Conflicts of Interest: The authors declare no conflict of interest.

\section{References}

1. Maurya, A.; Singh, A.K.; Mishra, G.; Kumari, K.; Rai, A.; Sharma, B.; Kulkarni, G.T.; Awasthi, R. Strategic use of nanotechnology in drug targeting and its consequences on human health: A focused review. Interv Med Appl Sci 2019, 11, 38-54.

2. Pérez-Herrero, E.; Fernández-Medarde, A. Advanced targeted therapies in cancer: Drug nanocarriers, the future of chemotherapy. European Journal of Pharmaceutics and Biopharmaceutics 2015, 93, 52-79.

3. Naz, S.; Shamoon, M.; Wang, R.; Zhang, L.; Zhou, J.; Chen, J. Advances in Therapeutic Implications of Inorganic Drug Delivery Nano-Platforms for Cancer. Int J Mol Sci 2019, 20, 965.

4. Vallet-Regi, M.; Tamanoi, F. Chapter One - Overview of Studies Regarding Mesoporous Silica Nanomaterials and Their Biomedical Application. In The Enzymes, Tamanoi, F., Ed. Academic Press: 2018; Vol. 43, pp. 1-10.

5. Patel, S.; Kim, J.; Herrera, M.; Mukherjee, A.; Kabanov, A.V.; Sahay, G. Brief update on endocytosis of nanomedicines. Advanced Drug Delivery Reviews 2019, 144, 90-111.

6. Maeda, H.; Wu, J.; Sawa, T.; Matsumura, Y.; Hori, K. Tumor vascular permeability and the EPR effect in macromolecular therapeutics: a review. Journal of Controlled Release 2000, 65, 271-284.

7. Boateng, F.; Ngwa, W. Delivery of Nanoparticle-Based Radiosensitizers for Radiotherapy Applications. Int J Mol Sci 2019, 21, 273.

8. $\quad$ K. Bakht, M.; Sadeghi, M.; Pourbaghi-Masouleh, M.; Tenreiro, C. Scope of Nanotechnology-based Radiation Therapy and Thermotherapy Methods in Cancer Treatment. Current Cancer Drug Targets 2012, 12, 998-1015.

9. Matsumoto, K.; Saitoh, H.; Doan, T.L.H.; Shiro, A.; Nakai, K.; Komatsu, A.; Tsujimoto, M.; Yasuda, R.; Kawachi, T.; Tajima, T., et al. Destruction of tumor mass by gadolinium-loaded nanoparticles irradiated with monochromatic X-rays: Implications for the Auger therapy. Sci Rep 2019, 9, 1327513275 .

10. Auger, P. The Auger effect. Surface Science 1975, 48, 1-8.

11. Rackwitz, T.; Debus, J. Clinical applications of proton and carbon ion therapy. Seminars in Oncology 2019, 46, 226-232.

12. Bushberg, J. The Essential Physics of Medical Imaging; Williams \& Wilkins: 1994.

13. Burattini, E.; Gambaccini, M.; Indovina, P.L.; Pocek, M.; Simonetti, G. [Synchrotron radiation: a new source in x-ray mammography]. Radiol Med 1992, 84, 181-188.

14. Lewis, R. Medical applications of synchrotron radiation x-rays. Physics in Medicine and Biology 1997, 42, 1213-1243.

15. Pole, D.; Popovic, K.; Williams, M. Contrast imaging with a monochromatic $x$-ray scanner; SPIE: 2008. 
16. Lawaczeck, R.; Arkadiev, V.; Diekmann, F.; Krumrey, M. Monochromatic X-rays in digital mammography. Invest Radiol 2005, 40, 33-39.

17. Martin, R.F.; Feinendegen, L.E. The quest to exploit the Auger effect in cancer radiotherapy - a reflective review. Int J Radiat Biol 2016, 92, 617-632.

18. Yokoya, A.; Ito, T. Photon-induced Auger effect in biological systems: a review. Int J Radiat Biol 2017, 93, 743-756.

19. Fairchild, R.G.; Brill, A.B.; Ettinger, K.V. Radiation enhancement with iodinated deoxyuridine. Invest Radiol 1982, 17, 407-416.

20. Tamura, K.; Ohko, Y.; Kawamura, H.; Yoshikawa, H.; Tatsuma, T.; Fujishima, A.; Mizuki, J. X-ray induced photoelectrochemistry on TiO2. Electrochim Acta 2007, 52, 6938-6942.

21. Liong, M.; Lu, J.; Kovochich, M.; Xia, T.; Ruehm, S.G.; Nel, A.E.; Tamanoi, F.; Zink, J.I. Multifunctional inorganic nanoparticles for imaging, targeting, and drug delivery. ACS Nano 2008, 2, 889-896.

22. Yanes, R.E.; Tamanoi, F. Development of mesoporous silica nanomaterials as a vehicle for anticancer drug delivery. Ther Deliv 2012, 3, 389-404.

23. Gisbert-Garzaran, M.; Manzano, M.; Vallet-Regi, M. Mesoporous Silica Nanoparticles for the Treatment of Complex Bone Diseases: Bone Cancer, Bone Infection and Osteoporosis. Pharmaceutics 2020, 12 .

24. Kwon, S.; Singh, R.K.; Perez, R.A.; Abou Neel, E.A.; Kim, H.W.; Chrzanowski, W. Silica-based mesoporous nanoparticles for controlled drug delivery. J Tissue Eng 2013, 4, 2041731413503357.

25. Li, T.; Shi, S.; Goel, S.; Shen, X.; Xie, X.; Chen, Z.; Zhang, H.; Li, S.; Qin, X.; Yang, H., et al. Recent advancements in mesoporous silica nanoparticles towards therapeutic applications for cancer. Acta Biomater 2019, 89, 1-13.

26. Lu, J.; Liong, M.; Sherman, S.; Xia, T.; Kovochich, M.; Nel, A.E.; Zink, J.I.; Tamanoi, F. Mesoporous Silica Nanoparticles for Cancer Therapy: Energy-Dependent Cellular Uptake and Delivery of Paclitaxel to Cancer Cells. Nanobiotechnology 2007, 3, 89-95.

27. Lu, J.; Liong, M.; Li, Z.; Zink, J.I.; Tamanoi, F. Biocompatibility, biodistribution, and drug-delivery efficiency of mesoporous silica nanoparticles for cancer therapy in animals. Small 2010, 6, 1794-1805.

28. Croissant, J.; Cattoen, X.; Man, M.W.; Gallud, A.; Raehm, L.; Trens, P.; Maynadier, M.; Durand, J.O. Biodegradable ethylene-bis(propyl)disulfide-based periodic mesoporous organosilica nanorods and nanospheres for efficient in-vitro drug delivery. Adv Mater 2014, 26, 6174-6180.

29. Maggini, L.; Cabrera, I.; Ruiz-Carretero, A.; Prasetyanto, E.A.; Robinet, E.; De Cola, L. Breakable mesoporous silica nanoparticles for targeted drug delivery. Nanoscale 2016, 8, 7240-7247.

30. Prasetyanto, E.A.; Bertucci, A.; Septiadi, D.; Corradini, R.; Castro-Hartmann, P.; De Cola, L. Breakable Hybrid Organosilica Nanocapsules for Protein Delivery. Angew Chem Int Ed Engl 2016, 55, 3323-3327.

31. Lu, N.; Fan, W.; Yi, X.; Wang, S.; Wang, Z.; Tian, R.; Jacobson, O.; Liu, Y.; Yung, B.C.; Zhang, G., et al. Biodegradable Hollow Mesoporous Organosilica Nanotheranostics for Mild Hyperthermia-Induced Bubble-Enhanced Oxygen-Sensitized Radiotherapy. ACS Nano 2018, 12, 1580-1591.

32. Croissant, J.G.; Fatieiev, Y.; Julfakyan, K.; Lu, J.; Emwas, A.H.; Anjum, D.H.; Omar, H.; Tamanoi, F.; Zink, J.I.; Khashab, N.M. Biodegradable Oxamide-Phenylene-Based Mesoporous Organosilica Nanoparticles with Unprecedented Drug Payloads for Delivery in Cells. Chemistry 2016, 22, 1480614811.

33. Mai, N.X.D.; Birault, A.; Matsumoto, K.; Ta, H.K.T.; Intasa-Ard, S.G.; Morrison, K.; Thang, P.B.; Doan, T.L.H.; Tamanoi, F. Biodegradable Periodic Mesoporous Organosilica (BPMO) Loaded with 
Daunorubicin: A Promising Nanoparticle-Based Anticancer Drug. ChemMedChem 2020, 10.1002/cmdc.201900595.

34. Mehta, G.; Hsiao, A.Y.; Ingram, M.; Luker, G.D.; Takayama, S. Opportunities and challenges for use of tumor spheroids as models to test drug delivery and efficacy. J Control Release 2012, 164, 192-204.

35. Luo, Y.; Wang, C.; Hossain, M.; Qiao, Y.; Ma, L.; An, J.; Su, M. Three-dimensional microtissue assay for high-throughput cytotoxicity of nanoparticles. Anal Chem 2012, 84, 6731-6738.

36. Lu, H.; Stenzel, M.H. Multicellular Tumor Spheroids (MCTS) as a 3D In Vitro Evaluation Tool of Nanoparticles. Small 2018, 14, e1702858.

37. Eggl, E.; Schleede, S.; Bech, M.; Achterhold, K.; Loewen, R.; Ruth, R.D.; Pfeiffer, F. X-ray phasecontrast tomography with a compact laser-driven synchrotron source. Proc Natl Acad Sci U S A 2015, $112,5567-5572$.

38. Biston, M.C.; Joubert, A.; Adam, J.F.; Elleaume, H.; Bohic, S.; Charvet, A.M.; Esteve, F.; Foray, N.; Balosso, J. Cure of Fisher rats bearing radioresistant F98 glioma treated with cis-platinum and irradiated with monochromatic synchrotron X-rays. Cancer Res 2004, 64, 2317-2323.

39. Hainfeld, J.F.; Ridwan, S.M.; Stanishevskiy, Y.; Panchal, R.; Slatkin, D.N.; Smilowitz, H.M. Iodine nanoparticles enhance radiotherapy of intracerebral human glioma in mice and increase efficacy of chemotherapy. Sci Rep 2019, 9, 4505.

40. Barth, R.F.; Vicente, M.G.; Harling, O.K.; Kiger, W.S., 3rd; Riley, K.J.; Binns, P.J.; Wagner, F.M.; Suzuki, M.; Aihara, T.; Kato, I., et al. Current status of boron neutron capture therapy of high grade gliomas and recurrent head and neck cancer. Radiat Oncol 2012, 7, 146.

41. Suzuki, M.; Kato, I.; Aihara, T.; Hiratsuka, J.; Yoshimura, K.; Niimi, M.; Kimura, Y.; Ariyoshi, Y.; Haginomori, S.; Sakurai, Y., et al. Boron neutron capture therapy outcomes for advanced or recurrent head and neck cancer. J Radiat Res 2014, 55, 146-153.

42. Nedunchezhian, K.; Aswath, N.; Thiruppathy, M.; Thirugnanamurthy, S. Boron Neutron Capture Therapy - A Literature Review. J Clin Diagn Res 2016, 10, ZE01-ZE04.

43. Suzuki, M. Boron neutron capture therapy (BNCT): a unique role in radiotherapy with a view to entering the accelerator-based BNCT era. Int J Clin Oncol 2020, 25, 43-50.

44. Achilli, C.; Grandi, S.; Ciana, A.; Minetti, G. Bnct and Nanoparticles: A Long Way to a Routine Clinical Method. . Int J Med Nano Res 2015, 2.

45. Barth, R.F.; Mi, P.; Yang, W.L. Boron delivery agents for neutron capture therapy of cancer. Cancer Commun 2018, 38.

46. Shih, J.L.A.; Brugger, R.M. Gadolinium as a Neutron-Capture Therapy Agent. Medical Physics 1992, 19, 733-744.

47. Cirrone, G.A.P.; Manti, L.; Margarone, D.; Petringa, G.; Giuffrida, L.; Minopoli, A.; Picciotto, A.; Russo, G.; Cammarata, F.; Pisciotta, P., et al. First experimental proof of Proton Boron Capture Therapy (PBCT) to enhance protontherapy effectiveness. Sci Rep 2018, 8, 1141. 